\title{
Consideraciones referente a la posibilidad de introducir la dosis fraccionada de la vacuna antipoliomielitis inactivada en el calendario de Inmunizaciones del Niño Latinoamericano
}

\author{
Antonio Arbo', Luiza Helena Falleiros-Arlant', Eduardo L. López', José Brea del Castillo', \\ Celia Martínez de Cuellar', Gabriela Moreno', Roger Rolón, Javier Cerda', \\ Salim Eguiazú y grupo de estudio de la Sociedad Paraguaya de Pediatría
}

\section{Remarks on the possibility of the introduction of fractionated dose of the inactivated poliomyelitis vaccine in the Latin American Child Immunization Schedule}

As last notified case of poliomyelitis due to wild poliovirus type 2 was 1999, in 2012, the Strategic Advisory Group of Experts on Immunization (SAGE) of the World Health Organization (WHO) recommended the withdrawal of the type 2 component of oral polio vaccine (OPV) and the introduction of bivalent OPV (bOPV) in all countries by 2016 . WHO recommended also that the withdrawal should be preceded by the introduction of at least one dose of inactivated poliovirus vaccine (IPV) in routine immunization schedules. The introduction of IPV prior to the change of the bOPV in 2016 to trivalent OPV (tOPV) was based on the concept of ensuring that a substantial proportion of the population would be protected against type 2 polio after the removal of the type 2 OPV. However, the world's two producers of IPV (Bilthoven Biologicals and Sanofi) have faced problems in the production of this vaccine and therefore reported reduction in IPV global supply. In response to the possible shortage of IPV, the SAGE and Technical Adviser Group (TAG) of the Pan American Health Organization (PAHO), in the meeting of March 10, 2017, has urged that countries in the Latinamerican region should replace the routine administration of the full doses of polio inactivated vaccine (IPV-C) in the immunization schedule (administered by intramuscular route) by the administration of a fraction of the full dose in two shots by intradermal route (IPV-f). The possibility of this strategy was analyzed by leaders of opinions gathered by the call of the Paraguayan Pediatric Society with the support of the Latin American Society of Pediatric Infectious Diseases (SLIPE) and Latin American Association of Pediatrics (ALAPE). The results of the discussion are presented in this document.

\section{Introducción}

E 1 presente documento es el resultado de la reunión realizada en Asunción, Paraguay, los días 17 y 18 de noviembre de 2017. La mencionada reunión fue una iniciativa de la Sociedad Paraguaya de Pediatría, con el apoyo de la Sociedad Latinoamericana de Infectologia Pediátrica (SLIPE) y Asociación Latinoamericana de Pediatría (ALAPE) ante la recomendación del Grupo Técnico Asesor (GTA) de la Organización Panamericana de la Salud (OPS), del 10 de marzo de $2017^{1}$, de instar a los países de la región a substituir en el calendario de inmunización la administración habitual de la dosis completa de la vacuna inactivada anti-virus de la polio (IPV-C) (administrada por vía intramuscular) por la administración de una fracción de la dosis completa (1/5 de la dosis) por vía intradérmica (IPV-f). Esta recomendación surge en respuesta a la posibilidad de déficit en la producción y, por lo tanto, en la disponibilidad de la vacuna inactivada contra la poliomielitis (IPV) a nivel mundial, debido a que productores mundiales han enfrentado problemas en la producción de esta vacuna y que han comunicado varias reducciones en las ofertas mundiales, previéndose un desabastecimiento global de IPV que se intensificará y se mantendrá al menos hasta finales del $2018^{1,2}$.

\section{Situación actual de la poliomielitis en el mundo y en las Américas}

A finales de la década de los 80, la Asamblea Mundial de la Salud resolvió erradicar la poliomielitis, causa principal de parálisis irreversible (predominantemente en los miembros inferiores) en pediatría ${ }^{3}$. Desde entonces, el número de casos de infección sintomática producidos por el virus salvaje de la polio (WPV) ha disminuido
'Sociedad Latinoamericana de Infectología Pediátrica. Recibido: 7 de junio de 2018 Correspondencia a: Antonio Arbo Sosa antonioarbo@hotmail.com

Luiza Helena Falleiros-Arlant luizahfc@terra.com.br 
$>99,9 \%$, de unos 350.000 casos de polio estimados en el año 1988 a 37 casos notificados en tres países en el año 2016 y 22 casos en dos países (Afganistán y Paquistán) en 2017 y ocho casos hasta 29 de mayo de 2018, también en Afganistán y Paquistán ${ }^{4}$. En nuestro continente, en el año 1985, los países de las Américas establecieron la meta de erradicar la polio en la Región, observándose el último caso de polio en Las Américas en Junín, Perú, el 23 de agosto de $1991^{5}$. En el año 1994, la Región de las Américas fue certificada como libre de la circulación del $\mathrm{WPV}^{6}$. Esta disminución se logró principalmente mediante el uso de vacuna de virus polio oral trivalente (tOPV), que contiene virus vivos de la polio atenuados de los tipos 1,2 y $3^{2}$.

\section{Vacuna OPV y riesgo de parálisis fláccida}

La utilización de la vacuna OPVt no está libre de riesgos. La vacuna puede asociarse a enfermedad produciendo casos de poliomielitis por el virus relacionado a la vacuna (VAPP), pero además significantemente cualquiera de los componentes puede revertir su atenuación y causar parálisis, pudiendo observarse casos de polio por virus derivado de la vacuna, que ha revertido su calidad atenuada por mutación genética (VDPV). El riesgo de VAPP varía de pais a país en Latinoamérica, de 1 caso por cada 2,56 a 4,1 millones de dosis aplicadas ${ }^{7}$. La enfermedad causada por el virus mutante derivado de la vacuna tiene la misma clínica, gravedad y letalidad que la poliomielitis causada por el virus salvaje. Nuestro continente experimentó en el año 2001 un brote de VDPV, en la Isla La Española con 21 casos confirmados debido a la mutación del serotipo 1 de la vacuna ${ }^{8}$. En las últimas décadas más de 500 casos de VDVP han sido reportados en el mundo. Casi todos los brotes de VDPV (97\%) en los últimos años han sido causados por un virus derivado de la vacuna OPV de tipo $2^{4}$, estimándose que por cada caso reportado 100 a 1.000 personas están eliminando en forma asintomática el virus en el ambiente? .

Aunque desde el año 2000, los Estados Unidos de América (E.U.A.) y posteriormente la mayoría de los países europeos han utilizado exclusivamente la vacuna IPV $^{10}$, que contiene los tres tipos de poliovirus (1, 2, 3), la gran mayoría de los países de América Latina, de África y Asia han continuado utilizando para la inmunización contra la enfermedad la vacuna OPVt hasta abril de 2016, aunque ya en el año 2014 la Sociedad Latinoamericana de Infectologia Pediátrica (SLIPE) y la Asociación Latinoamericana de Pediatría (ALAPE) habían recomendado la substitución de la OPV por la IPV dado que el riesgo de enfermedad por la vacuna supera al riesgo de enfermedad por la adquisición natural del virus salvaje de la polio ${ }^{11}$.

\section{Situación de la región de las Américas en cuanto a riesgo de introducir el virus de la polio importado}

La transmisión del virus salvaje de la polio continúa en 2018 en Afganistán y Pakistán ${ }^{4}$. Si no se detiene la transmisión en estos últimos reductos restantes, se podrían producir hasta 200.000 nuevos casos anuales en 10 años en todo el mundo ${ }^{12}$. Aunque la migración de esos países es muy baja en America Latina, la tasa de inmigración desde países africanos (principalmente Angola al Brasil) no es despreciable, lo que hace que esta región no está exenta del riesgo de la introducción en forma importada del virus de la poliomielitis ${ }^{13}$. En un ejercicio de análisis de riesgo de los países de la región de las Américas llevado a cabo por la OPS ${ }^{14}$ en el cual se han utilizado cuatro componentes que incluyen: 1) cobertura de inmunizaciones; 2) vigilancia de Parálisis Fláccida Aguda; 3) antecedente de brotes, o historia de circulación de virus derivado de vacuna y disponibilidad de plan de respuesta y 4) capacidad nacional de detectar importación de polio salvaje o eventos asociados a virus derivados de la vacuna, se ha construido una escala de riesgo para los países que va desde 0 (riesgo mínimo) a 10 (alto riesgo). Varios países de la Región se sitúan en un nivel de riesgo moderado o muy alto (en América del Sur, Venezuela), y en el Cono Sur exhiben niveles preocupantes de riesgo Brasil y Paraguay (Tabla 1). Esta situación obliga a que cualquier determinación que se realice referente a la estrategia de inmunización contra la polio debe ser profundamente meditada y analizada.

\section{Razones para la remoción del componente polio 2 de la vacuna OPVt e introducción de IPV en el esquema primario de vacunación}

El poliovirus salvaje de tipo 2 no ha circulado de forma natural desde el año 1999 (último caso en Aligarh, India), lo que obvia la necesidad del componente de tipo 2 de la vacuna ${ }^{2}$. Además, la presencia del componente de tipo 2 en la vacuna disminuye la respuesta inmune a los virus polio de tipo 1 y 3 y se requieren más dosis de OPVt para alcanzar los umbrales de inmunidad colectiva para estos dos serotipos en comparación con el número de dosis de la vacuna bivalente que contiene solamente los dos serotipos 1 y $3(\mathrm{OPVb})$ para alcanzar los mismos umbrales de inmunidad ${ }^{15}$.

La $65^{\circ}$ Asamblea Mundial de la Salud (mayo de 2012) aprobó la recomendación "completar la erradicación del virus de la polio como emergencia programática de la salud pública mundial"16. En la Formulación del Plan Estratégico para la erradicación de la poliomielitis y la fase final 2013-2018 para que el mundo esté libre de la 
poliomielitis, exhortó a interrumpir el uso de la vacuna oral contra la poliomielitis (OPVt) en todo el mundo, comenzando con el poliovirus de tipo 2 (OPV tipo 2), impulsando el cambio de la OPVt a la OPVb que contenga los serotipos 1 y $3^{2}$. Sin embargo, debido a la persistencia del riesgo de parálisis asociado al virus vacunal tipo 2 circulante o que pueda ser importado (riesgo que se estima va a persistir hasta cinco años después de la interrupción de la administración de la OPVt), se ha considerado necesaria que la población permanezca inmunizada contra el serotipo 2 de la Polio con un inmunógeno que contenga la forma inactivada del virus, que no se elimine y que no haya el riesgo de mutación en nivel intestinal. Por lo tanto los países que implementen la OPVb como una medida de mitigación de riesgos deben introducir al menos una dosis de la IPV ${ }^{16}$. La introducción de la IPV antes del cambio de la OPVt a la OPVb asegura que una proporción sustancial de la población esté protegida contra la poliomielitis de tipo 2 después del retiro de la OPV de tipo 2 y de esta manera se mitigan los riesgos de la reintroducción del tipo 2, al par de facilitar la erradicación de la poliomielitis al fortalecer la inmunidad a los tipos 1 y 3 .

\section{Propuesta de introducir IPV-f en substitución de IPV-C}

Cuando el The Polio Eradication \& Endgame Strategic Plan 2013-2018 fue socializado en el año 2013, ya se anticipaba el riesgo de déficit en el abastecimiento de la vacuna IPV para suplir la mayor demanda ${ }^{2}$. Ya en el año 2014 la producción de IPV había caído en $40 \%$ y se anticipaba que los problemas de producción persistirían más de cinco años, con posible mejoría recién en el 2020. En respuesta a esta situación, el grupo de expertos de OMS y OPS han sugerido que "los países consideren la adopción de dosis fraccionada de la vacuna polio inactivada via intradérmica" ${ }^{2,14,15}$. Esta recomendación-aunque no menciona taxativamente- es para países de América Latina, África y Asia y no para E.U.A. y Europa. El esquema de inmunización sugerido es de administrar las dos primeras dosis (de los 2 y 4 meses) con IPV-f y las siguientes con OPVb (Cuadro 1).

\section{Inmunogenicidad de la vacuna IPV-f}

Ante la recomendación, por parte de OPS/OMS como parte de la respuesta al desabastecimiento de la IPV, de uso de por vía intradérmica de una fracción de la dosis completa de la vacuna IPV, específicamente la quinta parte de la dosis de vacuna completa administrada por vía intramuscular, múltiples estudios han evaluado la inmunogenicidad de la IPV administrada en dosis frac-

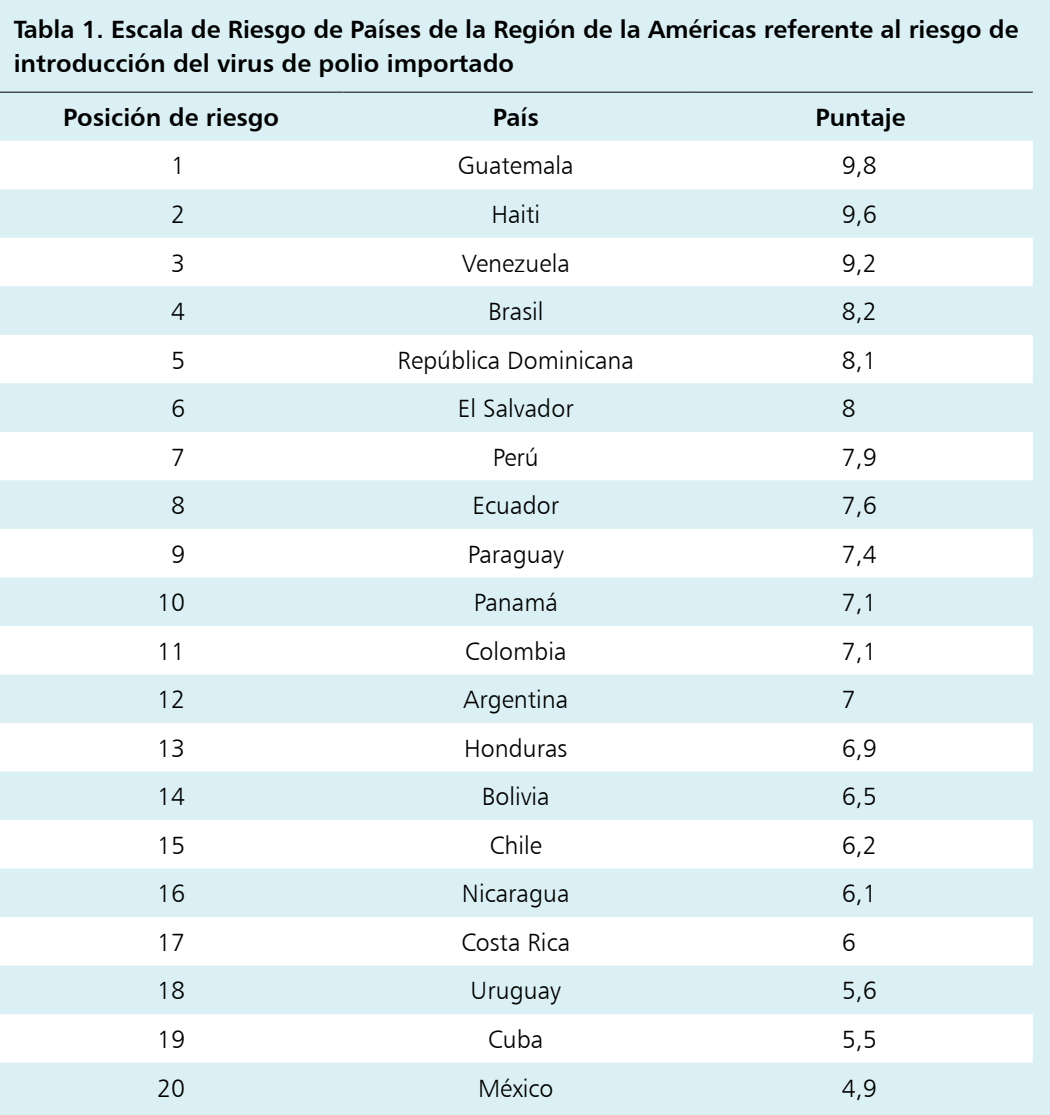

Fuente: PAHO. Technical Advisory Group on Vaccine-preventable Diseases (TAG). XXIV Meeting 12-14 July 2017

Cuadro 1. Esquema de vacunación anti-Polio propuesto por el GTA de la OPS

\begin{tabular}{lccccc}
\hline $\begin{array}{l}\text { Esquema de } \\
\text { vacunación }\end{array}$ & Básico & \multicolumn{2}{c}{ Refuerzos } \\
& $\mathbf{1}^{\text {a }}$ dosis & $2^{\text {a }}$ dosis & $3^{\text {a }}$ dosis & $1^{\text {er refuerzo }}$ & $2^{\circ}$ refuerzo \\
& IPV- $f$ & $I P V-f$ & bOPV & bOPV & bOPV \\
\hline
\end{tabular}

cionada por vía intradérmica (IPV-f) en comparación con la aplicación de IPV en dosis habitual o completa por vía intramuscular (IPV-C). Resik S. et $\mathrm{al}^{17}$, en un estudio de asignación aleatoria realizado en Cuba, han comparado la inmunogenicidad de IPV-f (una quinta parte de una dosis completa) administrada por vía intradérmica con un dispositivo especial (Bioejector 2000, Bioject) con la de IPV-C administrada por vía intramuscular, en 310 lactantes de 4 y 8 meses y han demostrado que tanto la seroconversión luego de la primera dosis como de la segunda dosis de IPV-f fueron significativamente menores para los tres serotipos. Con la primera dosis, la seroconversión es de IPV-f comparada con IPV-C para 
el serotipo 1 fue de $16,6 \%$ vs 46,4\% (p $<0,001)$, para el serotipo 2 de $47,1 \%$ vs $62,7 \%(p=0,008)$; y para el serotipo $3,14,6 \%$ vs $32 \%$ ( $p<0,001)$, Con la segunda dosis, la seroconversión de IPV-f comparada con IPV-C para el serotipo 1 fue de $92,4 \%$ vs $100 \%(p=0,01)$, para el serotipo $2,96,4 \%$ vs $100 \%(\mathrm{p}=0,41)$, y para el serotipo $3,91,8 \%$ vs $99 \%(p=0,018)$.

En un diseño diferente en cuanto a edad de administración de la vacuna, Mohammed AJ et al ${ }^{18}$, en un estudio realizado en Oman, patrocinado por la OMS, 373 niños fueron asignados al azar, en dos grupos, recibiendo uno de ellos $(\mathrm{n}=187$ niños) la dosis fraccionadas de vacuna IPV $(0,1 \mathrm{ml}$, que representa una quinta parte de una dosis completa) administrada por vía intradérmica con el uso de dispositivo especial (Bioejector 2000, Bioject) y el otro grupo $(n=186)$ dosis completas de vacuna administradas por vía intramuscular, a los 2, 4 y 6 meses, demostrando que las dosis fraccionadas de IPV administrada vía intradérmicamente a los 2, 4 y 6 meses, en comparación con dosis completas de IPV inducen niveles similares de seroconversión pero títulos significativamente más bajos. Así, la seroconversión a los 30 días después de completar el programa de tres dosis, para los serotipos 1, 2 y 3 de poliovirus fueron de $97,3 \%, 95,7 \%$ y $97,9 \%$, respectivamente, en el grupo de dosis fraccionadas, en tanto que la seroconversión fue del $100 \%$ a los 3 serotipos en el grupo de dosis completa $(\mathrm{p}=0,01$ para la comparación con respecto al poliovirus tipo 2 ; las diferencias con respecto a los poliovirus tipos 1 y 3 no fueron significativas). La mediana de los títulos de anticuerpos fueron igualmente significativamente más bajos en el grupo de dosis fraccionadas que en el de dosis completa $(\mathrm{p}<0,001$ para los tres serotipos de poliovirus) (Tabla 2 ).

Por su parte Cardona-Carlos $\mathrm{J}$ et al. ${ }^{19}$, en Filipinas administraron IPV-f aplicada por vía intradérmica con

Tabla 2. Tasas de seroconversión después de la administración de un programa de tres dosis de vacuna de poliovirus inactivada en forma fraccionada y mediana de títulos recíprocos de anticuerpos

\begin{tabular}{|c|c|c|c|}
\hline & \multicolumn{2}{|c|}{ \% de seroconversión } & \multirow[t]{2}{*}{ Valor $p$} \\
\hline & $\begin{array}{l}\text { Dosis fraccionada } \\
\qquad \mathbf{n}=187\end{array}$ & $\begin{array}{l}\text { Dosis completa } \\
n=186\end{array}$ & \\
\hline \multicolumn{4}{|c|}{ Pos-Dosis 3 (7 meses) } \\
\hline Polio 1 & $97,3 \%$ & $100 \%$ & 0,07 \\
\hline Polio 2 & $95,7 \%$ & $100 \%$ & 0,01 \\
\hline Polio 3 & $95,7 \%$ & $100 \%$ & 0,13 \\
\hline \multicolumn{4}{|c|}{ Título de Ac (7 meses) } \\
\hline Polio 1 & $228(144-287)$ & $(575-912)$ & $<0,001$ \\
\hline Polio 2 & $287(228-456)$ & $(912-1.149)$ & $<0,001$ \\
\hline Polio 3 & $362(287-456)$ & $\geq 1.448(\geq 1448-\geq 1.448)$ & $<0,001$ \\
\hline
\end{tabular}

jeringa y agujas de $13 \mathrm{~mm}, 30 \mathrm{G}$ o una dosis de IPV-C por vía IM a lactantes sanos a las 6,10 y 14 semanas y un refuerzo a los 15-18 meses de edad. Aunque los niveles de seroconversión después de tres dosis de IPV-f así como después del refuerzo fueron comparables a los que recibieron dosis de IPV-C, nuevamente los niveles de los títulos de media geométrica fueron claramente inferiores en todas las mediciones en el grupo que recibió IPV-f. Como en este estudio las muestras de sangre se tomaron después de la serie primaria y después del refuerzo, no se disponen de información de niveles de seroconversión ni de los títulos de media geométrica después de sólo dos dosis de IPV-f.

Resik S y cols. ${ }^{20}$, estudiaron la inmunogenicidad de la IPV-f administrada como refuerzo en un estudio controlado en adultos comparándolo con la IPV-C administrada por via intramuscular. Los porcentajes de seroconversión contra los tres serotipos fueron comparables, pero nuevamente los títulos de anticuerpos fueron inferiores en los que recibieron la IPV-f.

El estudio de Bibby $\mathrm{J}$ et $\mathrm{al}^{21}$, demuestra que en niños de 9-10 meses la respuesta es dependiente del dispositivo utilizado para la administración intradérmica de la vacuna y del tamaño de la pápula. La respuesta se correlaciona significantemente con el tamaño del habón que se obtiene con la administración intradérmica de la vacuna, lo que demuestra también que puede haber heterogeneidad en la administración en un estudio controlado de campo. Este aspecto puede acentuarse en el escenario real donde la capacitación del personal puede no ser uniforme y eficiente en todos los vacunatorios y no puede compararse al personal entrenado para estudios de investigación.

Dado que actualmente algunos países como Paraguay utilizan en la estrategia del cambio de vacuna tOPV a bO$\mathrm{PV}$ una sola dosis de IPV, se revisó la seroconversión con diferentes esquemas combinados de la IPV-C y la bOPV vs esquemas con todas las dosis de vacuna con IPV-C. En tal sentido, $\mathrm{O}^{\prime} \mathrm{Ryan} \mathrm{M}$ et $\mathrm{al}^{22}$, observaron que las tasas de seroconversión contra el poliovirus tipos dos fueron inferiores en el esquema secuencial que contenía una dosis de IPV-C seguida de dos dosis de bOPV (80,3\%), en comparación con esquemas dos dosis de IPV-C seguida de una dosis de bOPV (98,3\%) o el esquema con todas las dosis de IPV-C $(100 \%)(\mathrm{p}<0,0001)$. La significancia de tener una cohorte anual de $20 \%$ de niños (y probablemente mayor dado las coberturas vacunales subóptimas en Latinoamérica) sin seroconversión al poliovirus 2 es preocupante y debe analizarse adecuadamente.

\section{Efectos adversos}

Varios estudios han documentado una mayor proporción de efectos adversos con la utilización de la vía intradérmica, principalmente eritema e induración; 
Resik S. et $\mathrm{al}^{17}$, identificaron la presencia de eritema e induración en el sitio de aplicación de la vacuna IPV-f en 30 y 7\%, respectivamente, de los vacunados por vía intradérmica, con la primera dosis, significativamente mayor a lo observado en vacunados con la IPV-C en los que la presencia de eritema e induración en el sitio de aplicación se observaron en $2 \%(<0,001)$ y $1,3 \%$ $(\mathrm{p}=0,03)$, respectivamente. Con la segunda dosis de IPV-f se siguieron observando la misma frecuencia de efectos adversos. También Cardona-Carlos $\mathrm{J}$ et $\mathrm{al}^{19}$, en Filipinas identificaron una mayor frecuencia de eritema en pacientes vacunados con IPV-f, tanto en la primera como en la segunda dosis al compararlo con la IPV-C (69,5 vs $29,1 \%$ para la primera dosis y 38,4 vs $11,5 \%$ para la segunda dosis.

\section{Dificultades programáticas}

La OPS/OMS, en el documento de la Reunión ad-hoc virtual del GTA 2017, afirma "la administración de vacunas por vía ID es más difícil que por vía IM, es importante que los trabajadores de salud sean capacitados adecuadamente y supervisados de forma oportuna a fin de garantizar una vacunación segura. Para la introducción de un esquema de IPV-f, se deben considerar las actualizaciones de los sistemas de registro en los procesos de planificación, capacitación y supervisión”.

El mismo documento afirma "De acuerdo a la política de frascos abiertos de la OMS, la IPV en viales multidosis puede ser utilizada por un periodo de hasta 28 días. Para la aplicación de IPV-f, se debe utilizar una jeringa de 0,1 ml $27 \mathrm{G} 3 / 8$, la que es la misma utilizada para administrar la vacuna $B C G$ en algunos países. Hay países que administran la $B C G$ en jeringa de $0,05 \mathrm{ml} y$ por lo tanto, no tienen jeringa de 0,1 ml. Según datos del FR (Fondo Rotatorio), es muy probable que la jeringa de 0,1 ml para administración de la BCG no esté disponible en la mayoría de los países".

Uno de los desafíos adicionales en el eventual caso de adopción de la estrategia de vacunación de la IPV-f por vía intradérmica, es que la misma es más compleja que la administración de vacunas utilizando la vía intramuscular $^{23,24}$. Es necesario el entrenamiento del personal ya que no en todos los vacunatorios existe experiencia con vacunas de administración intradérmica (ej. BCG), la cual en la mayoría de los países de la Región se aplica en hospitales materno-infantiles y demanda mayor tiempo de aplicación. Los trabajadores de salud deben estar bien entrenados en la técnica, y la administración incorrecta puede conducir a eventos adversos o a menor tasa de seroconversión. En general la administración intradérmica se asocia a dificultad de determinar con precisión el volumen de vacuna administrada debido a la fuga en el sitio de la inyección ${ }^{21}$. La utilización de dispositivos especiales para la administración intradérmica puede ayudar a facilitar su implementación. Sin embargo, la utilización de estos dispositivos puede incrementar los costos en comparación a la administración intramuscular de la vacuna ${ }^{25}$.

En cuanto a la disponibilidad de dispositivos sin aguja (por ejemplo, inyectores de chorro de jeringa desechable) y dispositivos a base de aguja (por ejemplo, micro agujas huecas y adaptadores que se unen al centro de la aguja intradérmica y la jeringa), en Cuba se ha demostrado recientemente, que la seroconversión depende el dispositivo utilizado; así se ha observado comparando la respuesta inmune utilizando dos inyectores de jeringa desechables sin aguja (Tropis y Biojector 2000) con la utilización de aguja y jeringa de BCG, la misma fue similar contra los tres serotipos de poliovirus. Sin embargo, con un tercer inyector (Bioject) la respuesta inmune fue significativamente menor; con tasas de seroconversión para poliovirus tipo 2 de $22,6 \%$ en comparación con $49 \%{ }^{24}$.

Hay también otras preocupaciones referentes a la introduccion de la IPV-f. La aplicación de una vacuna intradérmica requiere personal con mayor capacitación y consume mas tiempo que una inyección intramuscular. Además, se está introduciendo una administración inyectable más, en un calendario ya repleto de administraciones inyectables en los lactantes. Además, se sabe por experiencia previa en America que las dosis adicionales de una determinada vacuna, especialmente inyectable, tienen el riesgo de una caída de la cobertura vacunal para la segunda dosis, quedándose estos niños con una sola dosis de la vacuna. Además, no se puede asegurar que también esta vacuna de aplicación intradérmica en dos dosis no va faltar.

\section{Discusión}

El grupo de trabajo, luego de revisar el reporte final de GTA de la OPS, la información disponible y del amplio debate, en relación al abordaje del desabastecimiento de la IPV en algunas áreas del mundo, que, sin embargo, no afecta a E.U.A. ni a Europa, definieron abordar cinco puntos:

- Inmunogenicidad de la IPV completa vs IPV fraccionada.

- Reactogenicidad-efectos adversos.

- Dificultades programáticas.

- Opciones alternativas.

- Aspectos éticos.

Concluyendo cuanto sigue:

\section{En cuanto a la inmunogenicidad de la IPV completa vs IPV fraccionada}

Referente a la IPV completa, estudios realizados en Latinoamérica han demostrado que esquemas de vacunación que incluyen una sola dosis de IPV seguida de dos 
dosis de bOPV, no generan una respuesta inmunogénica adecuada cuando se compara con un esquema de dos dosis de IPV-C seguida de una dosis de bOPV o esquemas de tres dosis de IPV-C.

Que los estudios realizados demuestran que la vacuna IPV utilizada en forma fraccionada (1/5 de la dosis completa) y administrada por vía intradérmica tiene una inmunogenicidad inferior a la obtenida con la primera dosis de IPV-C y similar con la segunda dosis.

Si bien no se ha definido la significancia de correlación de los niveles protectores de anticuerpos obtenidos con la IPV y durabilidad de la protección, los títulos de la media geométrica son superiores con la IPV-C en relación a los títulos obtenidos con la IPV-f. Este aspecto se considera importante ya que al no haber circulación natural del virus no se tendrán los estímulos antigénicos ambientales (booster).

Las coberturas sub-optimas, que se acentúan con la segunda dosis, en la mayoría de los países, podrían resultar en cohortes acumuladas de sujetos susceptibles, con el consecuente riesgo de desarrollar polio en situaciones de contacto posterior con algún virus salvaje o derivado de la vacuna.

Los estudios de investigación realizados son con grupos reducidos de niños y a corto plazo. Si bien tienen una validez interna, la que no está en discusión, no se puede probar la validez externa de ellos, ya que fueron realizados en condiciones controladas, y con distintos dispositivos de administración intradérmica que no son comparables entre sí y para la realización de los estudios el personal entrenado no puede compararse al personal entrenado a nivel poblacional. Este punto es importante porque no hay estudios que avalen que la administración técnicamente heterogénea produzca la misma respuesta inmune ni modifique la proporción de efectos adversos.

\section{En cuanto a reactogenicidad-efectos adversos}

Los estudios han demostrado que la administración de la IPV-f se ha acompañado de una mayor proporción de efectos adversos locales, principalmente induración y eritema local en $(30-60$ vs $<5 \%)$, comparado con la administración de la IPV por via intramuscular. Esto puede repercutir en las coberturas vacunales y generar opiniones contra las vacunas provenientes de los grupos anti-vacunas afectando a toda la campaña de vacunación en los países.

Se insta a los países a realizar una vigilancia exhaustiva a corto plazo de los eventos supuestamente atribuidos a la vacunación o inmunización (ESAVIS),

\section{En cuanto a las dificultades programáticas}

La aplicación intradérmica, conlleva a consideraciones logísticas, programáticas y operativas que requerirán una capacitación adecuada y supervisión continua a fin de ga- rantizar una vacunación segura. Debe anticiparse que, las dificultades programáticas de la aplicación intradérmica pueden afectar las coberturas actuales de vacunación.

Si bien el frasco multidosis de la vacuna IPV tiene una capacidad inmunogénica y bioactiva hasta 28 días después de abierto en condiciones controladas ${ }^{15}$, la utilización del biológico en terreno, puede llevar a contaminaciones relacionado a la manipulación y las consecuentes infecciones. Este riesgo no deberá minimizarse. Como ejemplo, en hospitales, aunque se sepa que la bioactividad de muchas medicaciones puede durar semanas es práctica cambiar los frascos antes de 7 días.

La estrategia de búsqueda activa de niños no vacunados, generalmente en comunidades alejadas, puede eventualmente realizarse con personal no adecuadamente capacitados, debido a las dificultades para capacitar al personal que trabaja principalmente en unidades periféricas o rurales de salud, como ocurre en algunas areas de América Latina.

La implementación de la vacuna IPV-f conlleva una inversión en la capacitación del personal, elaboración e impresión de manuales, que no siempre son implementados en forma óptima en terreno.

Se hace un llamado de atención, en lo que se refiere a la presente situación donde no se han realizado estudios del costo-efectividad, frente a un problema temporal de desabastecimiento. Por lo expuesto, la preparación para la introducción de la IPV-f, conlleva entre otros, a gastos para la preparación de la introducción de un nuevo esquema, que incluye la capacitación, preparación de materiales además de la adquisición del material de administración.

\section{En cuanto a opciones alternativas}

Se recomienda seguir las recomendaciones de SLIPE y ALAPE, ya sea con un esquema óptimo propuesto cuatro dosis de IPV-C (tres dosis en el esquema primario más un refuerzo) o esquemas con IPV-C en formulaciones combinadas con otros inmunogénicos indicadas en el programa de inmunización del país ${ }^{11}$. Es aceptable y no hay efectos adversos referente a que el niño vacunado con vacunas combinadas reciba más dosis de IPV-C que las recomendadas en el esquema primario; o un esquema alternativo, por ahora, mientras se usa la bOPV, que combine las dos o las tres primeras dosis de IPV-C seguidas por una o dos dosis de bOPV. Al mismo tiempo debe garantizarse el abastecimiento de las combinaciones vacunales con IPV.

Se exhorta a los países que sólo tienen una dosis de IPV-C, a actualizar sus esquemas vacunales, en la brevedad posible, con al menos dos dosis de IPV-C, teniendo en cuenta a la respuesta inmunogénica sub-óptima observada con los esquemas que incluyen una sola dosis.

Se insta a la OPS, a los ministerios de salud y a la industria farmacéutica, al diálogo y negociación de manera 
a incrementar la producción de la IPV-C y así evitar su desabastecimiento.

Finalmente, no recomendamos el uso de la IPV-f, en base a las evidencias científicas en cuanto a inmunogenicidad, frecuencia de efectos adversos y dificultades programáticas, y se insta a los países a analizar las opciones existentes de vacuna IPV-C, ya sea en frascos mono dosis, frascos multidosis o vacunas combinadas que pueden incorporarse a los esquemas de vacunación.

\section{En cuanto a los aspectos éticos}

El grupo de expertos no puede avalar ni recomendar la administración de un producto como es el caso de la IPV-f que no tiene indicación por parte del productor y no hay aprobación de la autoridad regulatoria de los países. Por lo tanto, las vacunas IPV-C usadas actualmente en los programas de vacunación de América Latina no tienen indicación para fraccionamiento.

Los estudios muestran respuesta inmunogénica de muy corto plazo, pero no hay estudios que demuestren que $1 / 5$ de la dosis aplicada de IPV-C provea de la misma protección a largo plazo. Existe el riesgo de dejar una cohorte de niños que no tendrán buena protección en el futuro y además de ser susceptibles, será una carga y preocupación extra para los países ya que esas cohortes deberán ser revacunadas.

Teniendo en cuenta los derechos humanos, y muy particular de los niños, con la utilización de la vía intradérmica, se está vulnerando el principio de protección al menor al exponerlo a una vía de administración con más efectos adversos en términos de dolor, induración y eritema. Además, el aspecto legal de protección del personal de salud es muy importante a la luz de que el uso de la vía intradérmica tiene más efectos adversos, lo que puede erróneamente atribuirse al personal de salud y disminuir la confianza en los mismos. Además, debido a los efectos adversos ocasionados por la aplicación por vía intradérmica en relación a la vía IM, se pone en riesgo la aplicación de dosis posteriores, comprometiendo las coberturas de vacunación.

Existe una discusión ética con respecto a la equidad, teniendo de que OPS/OMS está induciendo a que América Latina introduzca el uso de IPV-f, opción que no se está solicitando a países de Europa y E.U.A., generado una situación de precarización de esquemas óptimos de inmunización y con evidentes beneficios a los países centrales y generando una condición de inequidad en la salud pública mundial.

Finalmente, el grupo de expertos no recomienda en este momento el uso fraccionado de la IPV teniendo en cuenta a todo lo mencionado previamente en cuanto a inmunogenicidad, efectos adversos y dificultades programáticas, aspectos que pueden conllevar a brotes en un futuro, más aún cuando existen opciones de IPV disponibles al momento actual.

\section{Consideraciones finales}

El grupo de expertos hace un llamado a los países de América Latina para la toma de decisiones.

Si bien existe evidencia de inmunogenicidad de dos dosis de IPV-f, la evidencia, por sí sola no es suficiente para la toma de decisiones. En vacunas se debe considerar diversos aspectos, los cuales se relacionan con:

- Magnitud del problema.

- Beneficios vs efectos adversos.

- Protección a largo plazo.

- Costo-efectividad.

- Factibilidad de implementación.

- Disponibilidad del producto.

- Aceptación social.

- Seguridad del producto.

- Equidad.

- Aspectos legales y de regulatorios de la autoridad sanitaria.

- Aspectos éticos.

Por consiguiente, se requiere un análisis que involucre estas dimensiones para tomar la mejor decisión posible en una situación de desabastecimiento temporal.

\section{Integrantes del grupo de estudio de la Sociedad Paraguaya de Pediatría}

Rolón-Arámbulo Roger, Hospital Regional de Luque; Campuzano de Rolón Ana, Hospital de Clínicas, Universidad Nacional de Asunción; Lovera Dolores, Instituto de Medicina Tropical; Castro Héctor, Instituto de Previsión Social y Hospital de Niños Acosta-Ñú; Esquivel Ida, Sociedad Paraguaya de Pediatría; Nissen Julio, Sociedad Paraguaya de Pediatría; Araya Araya, Instituto de Medicina Tropical; Garcete Lidia, Hospital de Clínicas, Universidad Nacional de Asunción; Sánchez María José, Hospital Militar; Chirico Mirian, Hospital de Clínicas, Universidad Nacional de Asunción; Rodríguez Mónica, Instituto de Previsión Social; Bogado Norma, Hospital de Clínicas, Universidad Nacional de Asunción; Mir Ramón, Hospital de Clínicas, Universidad Nacional de Asunción; Olmedo Raúl, Sociedad Paraguaya de Pediatría; Flecha Claudia, Instituto de Previsión Social; Arrechea Ana, Hospital Materno-Infantil San Pablo. 


\section{Referencias bibliográficas}

1.- Pan American Health Organization. 2nd Ad-hoc Meeting of the Technical Advisory Group on Vaccine-preventable Diseases. 10 March 2017 Washington, DC, USA.

2.- World Health Organization. The Global Polio Eradication Initiative. Polio eradication and endgame strategic plan 2013-2018. Geneva, Switzerland; 2013.

3.- Organizacion Mundial de la Salud. $41^{\circ}$ Asamblea Mundial de la Salud. Ginebra, 2-13 de mayo de 1988. WHA41/1988/REC/1.

4.- World Health Organization. Global Polio Erradication Initiative. Available at: http:// polioeradication.org/polio-today/polio-now/ this-week/. (Acceso el 29 de mayo de 2018).

5.- Pan American Health Organization. Expanded Program on Immunization in the Americas. EPI newsletter, Bull Pan Am Health Organ. 14 ${ }^{\mathrm{a}}$ ed. Washington (DC); 1992.

6.- Pan American Health Organization. Expanded Program on Immunization in the Americas. EPI newsletter, Bull Pan Am Health Organ. 16 ${ }^{\mathrm{a}}$ ed. Washington D.C.; 1994.

7.- Landaverde J M, Trumbo S P, DanovaroHolliday M C, Cochi S E, Gandhi R, et al. Vaccine-associated paralytic poliomyelitis in the post elimination era in Latin America and the Caribbean, 1992-2011. J Infect Dis 2014; 209: 1393-402. doi: 10.1093/infdis/jit602

8.- Kew O, Morris-Glasgow V, Landaverde J M, Burns C, Shaw J, Garib Z, et al. Outbreak of poliomyelitis in Hispaniola associated with circulating type 1 vaccine-derived poliovirus. Science 2002; 296: 356-9. DOI:10.1126/ science. 1068284.

9.- Centers for Disease Control and Prevention. MMWR Morb Mortal Wkly Rep. Update on vaccine-derived polioviruses-worldwide, April 2011-June 2012. 2012; 61 (37); 741-6.

10.- CDC. Poliovirus prevention in the United States: updated recommendations of the
Advisory Committee on Immunization Practices (ACIP). MMWR Morb Mortal Wkly Rep 2000; 49 (No. RR-5).

11.- Falleiros-Arlant L H, Avila-Agüero M L, Brea del Castillo J, Mariño C. El desafío del cambio de la vacuna inactivada contra poliomielitis en América Latina. Declaración de la Sociedad Latinoamericana de Infectología Pediátrica (SLIPE). Rev Chilena Infectol 2014; 31: 590-96. http://dx.doi.org/10.4067/S071610182014000500012.

12.- Organización Mundial de la Salud. Poliomielitis. http://www.who.int/mediacentre/ factsheets/fs114/en/. (Acceso el 02 de junio de 2018).

13.- Wallace G S, Seward J F, Pallansch M A. Interim CDC guidance for polio vaccination for travel to and from countries affected by wild poliovirus. MMWR Morb Mortal Wkly Rep 2014; 63: 591-4.

14.- PAHO. Technical Advisory Group on Vaccinepreventable Diseases (TAG). XXIV Meeting 12-14 July 2017.

15.- PAHO. Background and Technical Rationale for introduction of one dose of inactivated polio vaccine (IPV) in routine immunization schedule. January 2015.

16.- World Health Organization. Poliomyelitis: intensification of the global eradication initiative. Geneva, Switzerland: World Health Organization; 2012.

17.- Resik S, Tejeda A, Sutter R W, Díaz M, Sarmiento L, Alemañi N, et al. Priming after a fractional dose of inactivated poliovirus vaccine. N Engl J Med 2013; 368: 416-24.

18.- Mohammed A, AlAwaidy S, Bawikar S, Kurup P J, Elamir E, Shaban M M, et al. Fractional doses of inactivated poliovirus vaccine in Oman. N Engl J Med 2010; 362: 2351-9. doi: 10.1056/NEJMoa0909383.

19.- Cardona-Carlos J, Vidor E, Bonnet M C. Randomized controlled study of fractional doses of inactivated poliovirus vaccine administered intradermally with a needle in the Philippines. Intern J Infect Dis 2012; 16: e110-6.

20.- Resik S, Tejeda A, Díaz M, Okayasu H, Sein C, Molodecky N A, et al. Boosting immune responses following fractional-dose inactivated poliovirus vaccine: a randomized, controlled trial. J Infect Dis 2017: 215; 175-82. doi: 10.1093/infdis/jiw492.

21.- Bibby J, Saidu Y, Umesi A, MonekeAnyanwoke N, Bashorun A O, Badjie Hydara $\mathrm{M}$, et al. The immunogenicity of fractional intradermal doses of the inactivated poliovirus vaccine is associated with the size of the intradermal fluid bleb. Clin Infect Dis 2017; 65: 851-4. DOI: 10.1093/cid/cix381.

22.- O'Ryan M, Bandyopadhyay A S, Villena R, Espinoza M, Novoa J, Weldon W C, et al. Inactivated poliovirus vaccine given alone or in a sequential schedule with bivalent oral poliovirus vaccine in Chilean infants: a randomised, controlled, open-label, phase 4, non-inferiority study. Lancet Infect Dis 2015; 15: 1273-82. DOI: https://doi.org/10.1016/ S1473-3099(15)00219-4.

23.- Okayasu H, Sein C, Chang Blanc D, González A R, Zehrung D, Jarrahian C, et al. Intradermal administration of fractional doses of inactivated poliovirus vaccine: a dose-sparing option for polio immunization. J Infect Dis 2017; 216 (S1): S161-S67. doi: 10.1093/infdis/jix038

24.- Hickling J K, Jones K R, Friede M, Zehrung D, Chenc D, Kristensenc D. Intradermal delivery of vaccines: potential benefits and current challenges. Bull World Health Organ 2011; 89: 221-6. doi: 10.2471/BLT.10.079426.

25.- Griffiths U K, Santos A C, Nundy N, Jacoby E, Matthias D. Incremental costs of introducing jet injection technology for delivery of routine childhood vaccinations: comparative analysis from Brazil, India, and South Africa. Vaccine 2011; 29: 969-75. doi: 10.1016/j. vaccine. 2010.11 .038 